EPJ Web of Conferences 113, 04008 (2016)

DOI: $10.1051 /$ epjconf/ 201611304008

(C) Owned by the authors, published by EDP Sciences, 2016

\title{
Few-Nucleon Research at TUNL: Probing Two- and Three- Nucleon Interactions with Neutrons
}

\author{
C.R. Howell ${ }^{1, a}$, W. Tornow ${ }^{1, b}$, and H. Witała ${ }^{2, c}$ \\ ${ }^{1}$ Department of Physics, Duke University and Triangle Universities Nuclear Laboratory, Durham, NC, USA \\ ${ }^{2} \mathrm{M}$. Smoluchowski Institute of Physics, Jagiellonian University, Cracow, Poland
}

\begin{abstract}
The central goal of few-nucleon research at the Triangle Universities Nuclear Laboratory (TUNL) is to perform measurements that contribute to advancing ab-initio calculations of nuclear structure and reactions. The program aims include evaluating theoretical treatments of few-nucleon reaction dynamics through strategically comparing theory predictions to data, determining properties of the neutron-neutron interaction that are not accessible in two-nucleon reactions, and searching for evidence of longrange features of three-nucleon interactions, e.g., spin and isospin dependence. This paper will review studies of three- and four-nucleon systems at TUNL conducted using unpolarized and polarized neutron beams. Measurements of neutron-induced reactions performed by groups at TUNL over the last six years are described in comparison with theory predictions. The results are discussed in the context of the program goals stated above. Measurements of vector analyzing powers for elastic scattering in $A=3$ and $A=4$ systems, differential cross sections for neutron-deuteron elastic scattering and neutrondeuteron breakup in several final-state configurations are described. The findings from these studies and plans for the coming three years are presented in the context of worldwide activities in this front, in particular, research presented in this session.
\end{abstract}

\section{Introduction}

The theoretical treatment of few-nucleon systems and light nuclei using realistic models of interactions between nucleons provides a formalism bridge for connecting phenomena in nuclear structure and reactions to quantum chromodynamics (QCD). Studies of bound-state properties and reaction dynamics in these systems allow for assessments of two- and three-nucleon force models over a wide range of phase space. The theory tool kit includes rigorous few-nucleon calculations, ab-initio structure calculations of light nuclei, phenomenological nuclear potential models, effective field theory calculations and lattice QCD computations. Studies of three- and four-nucleon systems at TUNL are discussed with emphasis on measurements sensitive to long-range three-nucleon interactions and to features of the nucleon-nucleon (NN) interaction that are not accessible using two-nucleon systems.

The mechanism by which the residual strong nuclear force is produced by the color interactions in QCD is one of nature's great mysteries. Because hadrons are color neutral the static strong force

\footnotetext{
a e-mail: howell@tunl.duke.edu

be-mail: tornow@tunl.duke.edu

ce-mail: ufwitala@cyf-kr.edu.pl
} 


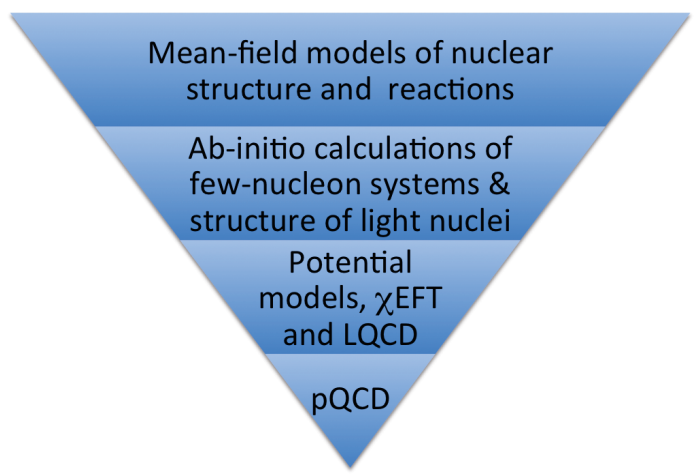

Figure 1. Schematic diagram for coherent theoretical treatment of nuclear systems starting from high energies where perturbative QCD can be applied going to low-energy nuclear phenomena where mean-field potential models are most efficient.

between nucleons is zero. Therefore, the strong nuclear force must be of a dynamical nature similar to Van der Waals interactions between charge-neutral atoms or molecules. For example, the spatial features of the NN potential are qualitatively similar to the London dispersive potential [1], which is due to the induced polarization of the atomic electron cloud relative to the nucleus. In this analogy the NN interaction is associated with the distortion of the pion cloud about the massive core. Qualitatively both potentials are attractive with effective ranges of distances several times the diameter of the interacting bodies and are repulsive at distances where the objects overlap. The dynamical nature of the nuclear force provides for rich phenomena and complexity that makes analysis of the systems interesting and challenging.

Over the last decade substantial progress has been made on developing formalism and computational methods that contribute to having theoretically coherent descriptions of nuclear phenomena with origins in QCD. An ultimate goal would be to describe nuclear matter over wide distance and energy scales with a QCD Lagrangian. Achieving this aim will likely require work done over generations of scientists, similar to other grand challenges in science and technology. A schematic diagram is shown in Fig. 1 of a plausible hierarchy of organizing the theoretical treatments of nuclear systems spanning a variety of phenomena. The scheme starts at ultra high energies, where the most fundamental degrees of freedom are quarks and gluons, and progressively evolves in complexity. This diagram is intended only to represent coarse features that should be included in a coherent picture of strongly interacting matter. In this framework the unifying concept is the residual strong force between nucleons. At the top of the diagram, mean-field potentials that describe nuclear structure properties, collective motion of nuclei and nuclear reactions should be derived from residual strong interactions between nucleons. Ab-initio calculations of the structure of light nuclei [2-5] and fewnucleon reaction dynamics [6] enable refinement of two-nucleon and multi-nucleon interactions using effective degrees of freedom. Current theory tools for describing the strong nuclear force (two- and three-nucleon interactions) include semi-empirical potential models, e.g., Refs. [7-11], effective field theory formulations of two-nucleon $(2 \mathrm{~N})$ and three-nucleon $(3 \mathrm{~N})$ interactions $[12,13]$, and Lattice QCD (LQCD) calculations of few-nucleon systems, e.g., see Refs. [14, 15]. Descriptions of the collective properties of nucleons in terms of effective field theories, e.g., Ref. [16] and LQCD, e.g., Ref. [17], are steps toward bridging gaps between QCD and theoretical treatments of few-nucleon systems.

In this paper we discuss the applications of few-nucleon systems to probe features of three-nucleon interactions (3NIs) and to evaulate current formulations of the neutron-neutron $(n n){ }^{1} \mathrm{~S}_{0}$ interaction. The general approach is to use ab-initio calculations of few-nucleon systems or light nuclei to search for observables that are sensitive to $3 \mathrm{~N}$ force (3NF) effects or to the ${ }^{1} \mathrm{~S}_{0} n n$ interaction. Such theory studies are useful for guiding experimental efforts. However, experiment planning should not be 


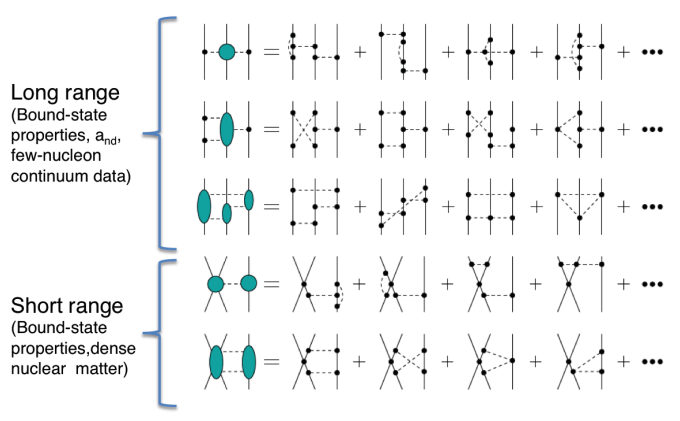

Figure 2. Diagrams of additional 3NI terms when increasing the $\chi$ EFT expansion from $\mathrm{N}^{2} \mathrm{LO}$ to $\mathrm{N}^{3} \mathrm{LO}$. This figure is from Ref. [13]. Expanding to $\mathrm{N}^{3} \mathrm{LO}$ results in increasing the number of interaction terms. However, there are no additional LECs when adding these higher order terms to the $3 \mathrm{NI}$ expansion. The diagrams can be divided into five topologies with the upper three lines contributing to the long-range component of the force, and the lower two lines providing short-range interactions.

strictly limited by them; there should be a purely exploratory component to provide opportunities for discovering effects not predicted by current theory. The sections that follow provide an overview of few-nucleon research at TUNL with focus on $3 \mathrm{NF}$ effects and the $n n$ interaction.

\section{Three-nucleon Interactions}

There is extensive evidence illustrating the importance of 3NIs in ab-initio theoretical treatment of nuclei. Examples include the triton-binding energy discrepancy $[8,10]$ and systematic studies of the ground and excited states of light nuclei using Monte-Carlo Green function simulations [2] and no-core shell model calculations [3-5]. Also, in the calculation of the nuclear equation of state, the energy per nucleon as a function of density, is highly sensitive to the inclusion of $3 \mathrm{NFs}$, especially at high nuclear densities relevant to the interior of neutron stars [18]. The indications for 3NF effects in low-energy scattering data are less definitive.

There are two types of 3NIs used in modern ab-initio nuclear calculations, semi-empirical potential, e.g., Refs. [10, 11] and effective field theory formulations, e.g., Refs. [12, 19-21]. The lowest order in Chiral Effective Field Theory ( $\chi$ EFT) where 3NI occur is next-to-next-to-leading or$\operatorname{der}\left(\mathrm{N}^{2} \mathrm{LO}\right)$. In this order there are two low-energy constants (LECs) associated with 3NI diagrams, often referred to as $\mathrm{c}_{D}$ and $\mathrm{c}_{E}$ [21]. These parameters can be determined by simultaneously fitting, e.g., the triton binding energy and ${ }^{2} \mathrm{a}_{n d}$. In this session, König [22] described calculations of the quartet proton-deuteron $(p d)$ scattering length as potentially being another observable for constraining the values of LECs in EFT interactions.

The additional non-vanishing 3NI diagrams added when increasing the expansion to next-to-nextto-next-to-leading order $\left(\mathrm{N}^{3} \mathrm{LO}\right)$ are shown in Fig. 2. The terms in $\mathrm{N}^{3} \mathrm{LO} 3 \mathrm{NIs}$ can be grouped according to their effective range. The features of the high-momentum components of nuclear wavefunctions and high-density nuclear matter, e.g., the interior of neutron stars as mentioned above, are sensitive to the short-range part of the $3 \mathrm{NI}$. The long-range component of the $3 \mathrm{NI}$, which is the theme of this session, can be probed by studying low-momentum components of nuclear wavefunctions via lowenergy few-nucleon reactions. Three presentations in this session are examples of such investigations. Sagara et al. [23] describe the energy dependence of the space-star (SST) in $p d$ breakup at proton energies below $20 \mathrm{MeV}$. They found that the ratio of the cross-section data to calculations is nearly constant with the data being about $8 \%$ lower than theory. In contrast, as shown in Figs. 3 and 4 , neutron-deuteron $(n d)$ data for the SST are about $30 \%$ larger than theory. The difference between the data and theory decreases with increasing neutron energy. In Fig. 3 the cross section is shown as a function of the arc-length $\mathrm{S}$ of the kinematical curve at incident nucleon energy of $13.0 \mathrm{MeV}$. The cross-section curves are Faddeev calculations using the CD-Bonn [7] NN potential and the TucsonMelbourne $3 \mathrm{~N}$ potential model [10]. The $p d$ data [26] plotted with the star symbols are about $10 \%$ 

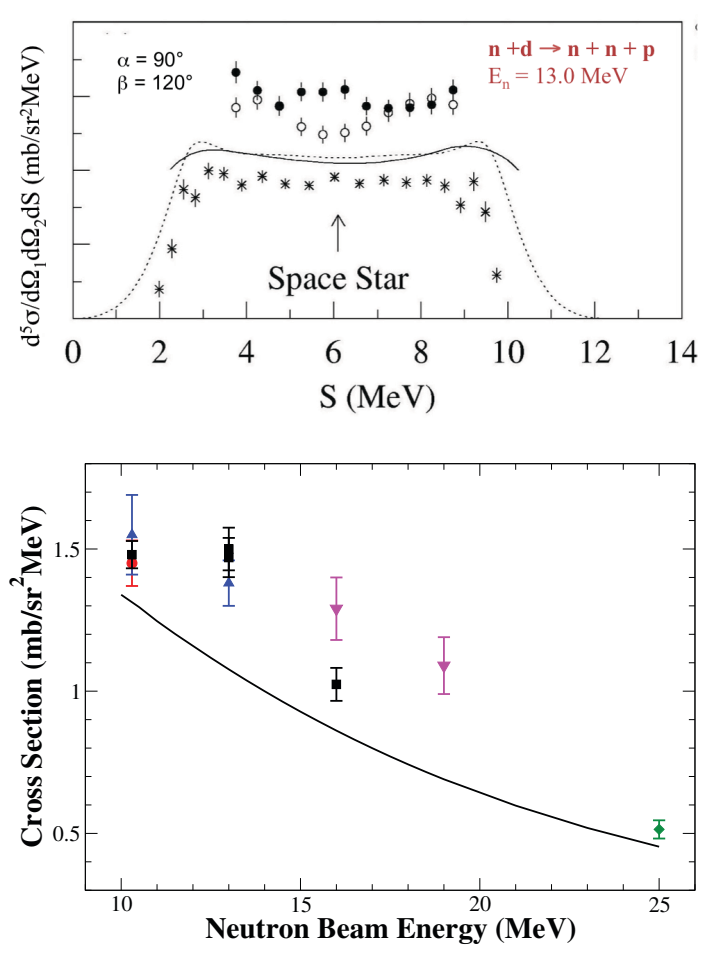

Figure 3. Cross sections for the space-star configuration in $n d$ breakup at an incident neutron energy of $13.0 \mathrm{MeV}$. The average angle of the detected neutrons are $\theta_{n 1}=\theta_{n 2}=50.3^{\circ}$ and $\phi_{12}=120^{\circ}$. This figure is from Ref. [24]. The data are $n d$ breakup from Ref. [24] (solid circles), nd breakup from Ref. [25] (open circles) and $p d$ breakup from Ref. [26] (stars). The curves are predictions of ab initio $n d$ breakup calculation with 3 NI. The dotted curve is a point-geometry calculation, and the solid curve is a simulation for the TUNL experiment [24].

Figure 4. Cross sections for the space-star configuration in $n d$ breakup as a function of an incident neutron energy. This figure is from Ref. [27]. The data are $n d$ breakup from Refs. [28-30] (squares), [27] (inverted triangles), [25, 31] (triangles), [32] (circles), and [33] (diamond).

lower than the theoretical predictions; the findings reported by Sagara at this conference [23] are consistent with these results. Long-range 3NIs that are not included in current calculations is a possible cause of the discrepancy between data and theory for the SST in nucleon-deuteron breakup.

Also in this session, calculations presented by Girlanda [34] illustrate that a solution to the longstanding " $\mathrm{A}_{y}$ puzzle" in $3 \mathrm{~N}$ and $4 \mathrm{~N}$ systems might be provided through the fitting flexibility enabled by the additional LECs for $3 \mathrm{NIs}$ in $\mathrm{N}^{4} \mathrm{LO}$ EFT interactions. Recent measurements made at TUNL of the vector analyzing power $\left(\mathrm{A}_{y}\right)$ for neutron- ${ }^{3} \mathrm{He}$ elastic scattering [35] are shown in Fig. 5. As in the case of the $3 \mathrm{~N}$ system, theory underpredicts the value of $\mathrm{A}_{y}(\theta)$ at the maximum for $\mathrm{n}^{3} \mathrm{He}$ elastic scattering. One approach to interpreting the $\mathrm{A}_{y}$ issue in the $4 \mathrm{~N}$ system is through the lens of isospin. Data for both mixed isospin $(T=0,1)$ and pure isospin $(T=1)$ states exist for proton scattering from ${ }^{3} \mathrm{H}$ and ${ }^{3} \mathrm{He}$, respectively. However, only data for the mixed isospin state $\left(\mathrm{n}^{3} \mathrm{He}\right)$ is available for neutron scattering. Esterline et al. [35] found that the energy dependence of the fractional difference between data and theory was similar for the mixed isospin $(\mathrm{T}=0,1)$ scattering systems of $\mathrm{p}^{3} \mathrm{H}$ and $\mathrm{n}^{3} \mathrm{He}$ and has a slope in the energy range studied opposite to that for the pure $\mathrm{T}=1 \mathrm{p}^{3} \mathrm{He}$ scattering. Measurements of $\mathrm{A}_{y}(\theta)$ for $\mathrm{n}^{3} \mathrm{H}$ elastic scattering are planned using the pulsed polarized neutron beam at TUNL. The goal of this experiment is to provide data that will complement the $\mathrm{p}^{3} \mathrm{He}(\mathrm{T}=1)$ data.

\section{Studies of the Neutron-neutron Interaction at TUNL}

In the two-nucleon system the values for the ${ }^{1} \mathrm{~S}_{0} \mathrm{NN}$ scattering length $\mathrm{a}_{N N}$ and effective range parameter $\mathrm{r}_{N N}$ are tightly constrained by limits on charge dependence and charge-symmetry violation in the strong NN interaction. This feature along with the high sensitivity of these parameters to the strength of the S-wave $\mathrm{NN}$ interaction provide means for probing few-nucleon systems and light nuclei for 


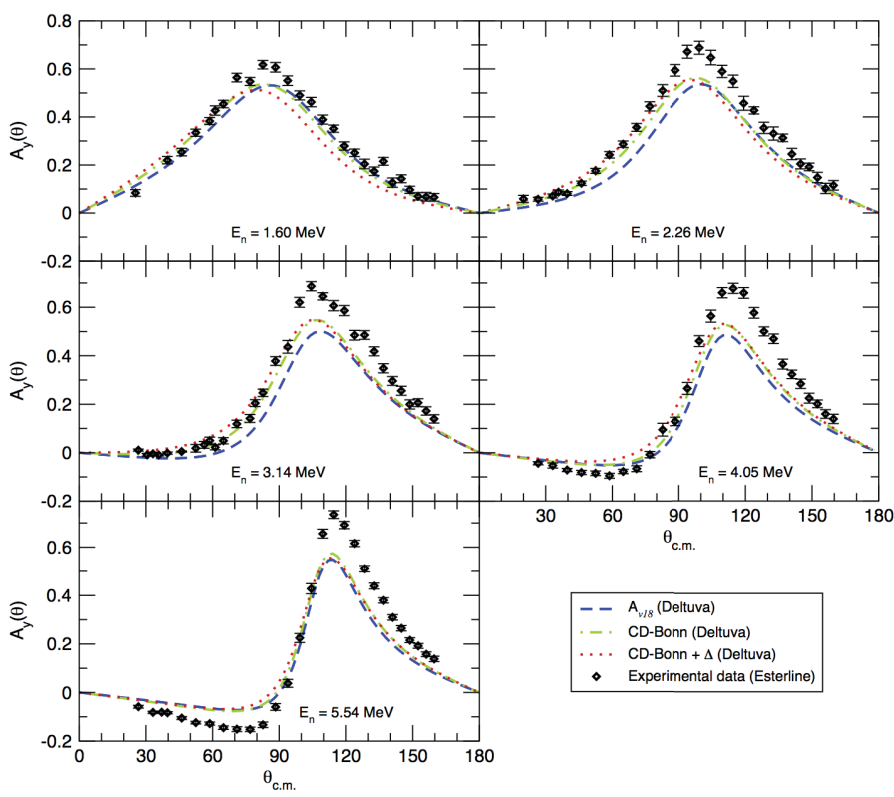

Figure 5. $\mathrm{A}_{y}(\theta)$ for ${ }^{-}{ }^{3} \mathrm{He}$ elastic scattering at several energies. This figure is adapted from Ref. [35]. The data are measurements from TUNL, and the curves are ab-initio calculations with the attributes indicated in the legend.

$3 \mathrm{NF}$ effects. For example, discrepancies in the values of $\mathrm{a}_{n n}$ from $\pi^{-} d$ capture data and those obtained from some $n d$ breakup measurements could indicate $3 \mathrm{NF}$ effects that are not included in current $n d$ breakup calculations [36]. A more recent observation of effects that could be due to 3NIs that are not included in current $n d$ calculations was reported by Witała and Glöckle in their analysis of $n n$ quasi-free scattering (QFS) cross-section data in $n d$ breakup [37]. The resolution of the about 20\% discrepancy between data and theory using current 3 NIs required substantial charge-symmetry breaking in the NN interactions beyond what is generally accepted; the value of $r_{n n}$ in their calculation was about $12 \%$ larger than allowed by charge symmetry.

There are two experiments underway at TUNL to further investigate these issues in the $n d$ continuum. One experiment is to measure the cross section for $n n$ QFS scattering in $n d$ breakup. This experiment is being carried out at two beam energies using the pulsed neutron beam facility at TUNL. Details and preliminary results for measurements at $10 \mathrm{MeV}$ are presented by Malone et al. [38] in this session. The other experiment is a measurement of the differential cross section for exclusive photodisintegration of ${ }^{3} \mathrm{H}$ in the kinematic region of the $n n$ FSI. These data will be used to determine a value of $\mathrm{a}_{n n}$ to investigate $3 \mathrm{NF}$ effects in tritium as probed by the photodisintegration reaction at gamma-ray energies below $20 \mathrm{MeV}$. The conceptual design of the experiment is described in the poster presentations by Friesen et al. [39] and Han et al. [40].

\section{Acknowledgements}

This work is supported in part by the U.S. Department of Energy under grant No. DE-FG02-97ER41033 and by the Polish National Science Center under Grant No. DEC-2013/10/M/ST2/00420.

\section{References}

[1] F. London, Transactions of the Faraday Society 33, 8-26 (1937); doi:10.1039/tf937330008b

[2] S.C. Pieper and V.R. Pandharipande, Annu. Rev. Nucl. Part. Sci. 51, 53 (2001) 
[3] P. Navratil et al., Phys. Rev. Lett. 99, 042501 (2007)

[4] P. Navratil et al., Few-Body Syst. 43, 129 (2008)

[5] A. Nogga, P. Navratil, B.R. Barrett and J.P. Vary, Phys. Rev. C 73, 064002 (2006)

[6] W. Glöckle et al., Phys. Rep. 274, 107 (1996)

[7] R. Machleidt, Phys. Rev. C 63, 024001 (2001)

[8] R.B. Wiringa, V.G.J. Stoks and R. Schiavilla, Phys. Rev. C 51, 38 (1995)

[9] V.G.J. Stoks, R.A.M. Klomp, C.P.F. Terheggen and J.J. de Swart, Phys. Rev. C 49, 2950 (1994)

[10] S.A. Coon, M.D. Scadron and B.R. Barrett, Nucl. Phys. A 242, 467 (1975)

[11] S.C. Pieper, V.R. Pandharipande, R.B. Wiringa and J. Carlson, Phys. Rev. C 64, 014001 (2001)

[12] R. Machleidt and D.R. Entem, Phys. Rep. 503, 1 (2011)

[13] E. Epelbaum, H.-W. Hammer and Ulf-G. Meissner, Rev. Mod. Phys. 81, 1773 (2009)

[14] E. Epelbaum, H Krebs, D. Lee and Ulf-G. Meissner, Phys. Rev. Lett. 106, 192501 (2011)

[15] S.R. Beane et al., Phys. Rev. Lett. 115, 132001 (2015)

[16] H.W. Griesshammer, J.A. McGovern, D.R. Phillips and G. Feldman, Prog. Part. Nucl. Phys. 67, 841 (2012)

[17] S. Beane et al., Phys. Rev. Lett. 113, 252001 (2014)

[18] P. Danielewicz, AIP Conf. Proc. 597, 24 (2001)

[19] R. Higa and M.R. Robilotta, Phys. Rev. C 68, 024004 (2003)

[20] R. Higa, M.R. Robilotta and C.A. da Rocha, Phys. Rev. C 69, 034009 (2004)

[21] E. Epelbaum et al., Phys. Rev. C 66, 064001 (2002)

[22] S. König and H. Hammer, Proc. Int. Conf. on Few-Body Problems in Physics (2015)

[23] K. Sagara et al., Proc. Int. Conf. on Few-Body Problems in Physics (2015)

[24] H.R. Setze et al., Phys. Lett. B 388, 229 (1996)

[25] J. Strate et al., Nucl. Phys. A 501, 51 (1989)

[26] G. Rauprich et al., Nucl. Phys. A 535, 313 (1991)

[27] A.H. Couture et al., Phys. Rev. C 85, 054004 (2012)

[28] H.R. Setze et al., Phys. Rev. C 71, 034006 (2005)

[29] A.S. Crowell, Ph.D. thesis, Duke University, 2001

[30] R.A. Macri, Ph.D. thesis, Duke University, 2004

[31] K. Gebhardt et al., Nucl. Phys. A 561, 232 (1993)

[32] M. Stephan et al., Phys. Rev. C 39, 2133 (1989)

[33] Z. Zhou et al., Nucl. Phys. A 684, 545c (2001)

[34] L. Girlanda et al., Proc. Int. Conf. on Few-Body Problems in Physics (2015)

[35] J.H. Esterline et al., Phys. Rev. Lett. 110, 152503 (2013)

[36] C.R. Howell, Proc. X Intern. Seminal. on Interactions of Neutrons with Nuclei, Dubna, Russia, ISINN-10 (2003), p. 71; arXiv:0805.1177

[37] H. Witała and W. Glöckle, Phys. Rev. C 83, 034004 (2011)

[38] R.C. Malone et al., Proc. Int. Conf. on Few-Body Problems in Physics (2015)

[39] F.Q.L. Friesen et al., Proc. Int. Conf. on Few-Body Problems in Physics (2015)

[40] Z. Han et al., Proc. Int. Conf. on Few-Body Problems in Physics (2015) 